\title{
Effectiveness case of Jidabokuippo (traditional Japanese Kampo medicine) on pain, venous thrombosis, and subcutaneous hematoma after percutaneous femoral catheterization in a hemodialysis patient
}

\author{
Kentaro Wada ${ }^{1 *}$, Haruhito A. Uchida ${ }^{2}$ and Yuko Wada ${ }^{3}$
}

\begin{abstract}
Background: The authors herein report a case of pain, femoral vein thrombosis, and inguinal subcutaneous hematoma caused by percutaneous femoral vascular catheterization in a hemodialysis (HD) patient complicated with vascular access occlusion. The pain was well-controlled without using analgesics such as non-steroidal antiinflammatory drugs (NSAIDs). The thrombosis and hematoma increased in size and was also treated by conservative treatment using traditional Japanese Kampo medicine (jidabokuippo: JDI) to resolve oketsu: a sign of microcirculating disorder. JDI improves the blood circulation in the affected lesion and alleviates contusion-induced blood flow and repairs blood flow without forming thrombosis. These catheter-related complications were successfully treated by JDI.

Case presentation: A 41-year-old man under HD was admitted because of permanent vascular access failure. He had a history of side effects of gastrointestinal bleeding associated with use of NSAIDs and accompanied with femoral vein thrombosis and inguinal subcutaneous hematoma caused by percutaneous femoral vascular catheterization. The hematoma increased in size and was treated using JDI. In the present case, pain, venous thrombosis, and subcutaneous hematoma surprisingly improved with use of JDI in addition to combination therapy with anticoagulant (warfarin potassium) and antibiotic (meropenem hydrate), without using analgesics such as NSAIDs.

Conclusions: The present case indicated as follows: JDI controls the pain associated with hematoma without analgesics such as NSAIDs; JDI also has an effect of improving the complications associated with venous thrombosis and subcutaneous hematoma caused by catheterization in HD patients safely. JDI, one of Kampo drugs, has few side effects and can be used as needed.
\end{abstract}

Keywords: Pain, Thrombosis, Hematoma, Catheterization, Hemodialysis, Jidabokuippo

\footnotetext{
* Correspondence: kyw620@hotmail.co.jp

${ }^{1}$ Division of Nephrology and Dialysis, Department of Internal Medicine,

Nippon Kokan Fukuyama Hospital, 1844, Tsunoshita, Daimon-cho, Fukuyama

City, Hiroshima 721-0927, Japan

Full list of author information is available at the end of the article
} 


\section{Background}

Percutaneous femoral catheterization is considered a quick and an easy access for emergency case under hemodialysis (HD) patient complicated with vascular access occlusion. However, it may cause severe and fatal complications such as infection, hematoma, deep vein thrombosis, and vessel perforation [1].

Conservative treatment of hematoma is acceptable if no hemodynamic dysfunction is present; no compartment syndrome is present; pain control is well-managed by analgesic drugs such as non-steroidal anti-inflammatory drugs (NSAIDs); and the hematoma does not increase in size.

The authors herein report a case of pain, femoral vein thrombosis, and inguinal subcutaneous hematoma caused by percutaneous femoral vascular catheterization. The thrombosis and hematoma increased in size and was successfully treated by conservative treatment using traditional Japanese Kampo medicine (jidabokuippo: JDI) to resolve oketsu: a sign of microcirculating disorder. The pain was also well-controlled without using analgesics. To our knowledge, no reports are available concerning the effectiveness of JDI for catheter-related complications in patients under HD.

\section{Case presentation}

A 41-year-old man (Japanese) under HD attributable to lupus nephritis for approximately 16 years with a history of vascular access trouble several times was admitted to our hospital because of permanent vascular access failure (day 1). Ultrasonography revealed that thrombosis obstructed the vascular access almost completely from the anastomosis of the brachial artery and artificial vessel toward the anastomosis of the brachial vein and artificial vessel. He had a risk factor of thrombosis with history of systemic lupus erythematosus (SLE) for 20 years. However, the laboratory data findings showed that anti-double strand DNA IgG antibody and anticardiolipine antibody remained negative, and 50\% hemolytic unit factor $(\mathrm{CH} 50), \mathrm{C} 3$, and $\mathrm{C} 4$ remained within normal range for recent 15 years. Thrombocytopenia was not observed. These data indicated that immunological activity of SLE was in remission.

The authors concluded the patient had no indication of thrombectomy nor percutaneous angioplasty. Percutaneous femoral vascular catheter was inserted (left) under ultrasonography guidance using the Seldinger technique because the patient had a history of carotid vein damage and subclavian vein stenosis. Since maintaining the appropriate blood flow rate was difficult, femoral vascular catheter was inserted again in the opposite (right) side. After the first HD session of catheterization, he complained of pain in the left groin; a hard, smooth, painful mass was palpable; and subcutaneous hemorrhage was also observed. The left groin was compressed with an elastic bandage.

On day 3, laboratory data findings showed elevation of d-dimer level $(13.2 \mu \mathrm{g} / \mathrm{mL})$ without any abnormality in blood coagulopathy, but no progression in the decrease of hemoglobin level. The patient also showed shivering and spiking fever $\left(39^{\circ} \mathrm{C}\right)$ and complained of continuous pain in the groin; therefore, the catheter was immediately removed. Contrast-enhanced computed tomography (CECT) revealed a thrombosis in the right side of the external iliac vein (Fig. 1, left panel) and a soft-tissue mass in the left side of the groin (Fig. 1, right panel), but revealed no pulmonary embolism.

Warfarin potassium was administered to maintain prothrombin time-international normalized ratio with the range from 1.5 to $2.5(2.0 \mathrm{mg} /$ day $)$. On the other hand, blood culture test was positive (Staphylococcus aureus), and laboratory data findings showed elevation of both white blood cell count $(12,400 / \mu \mathrm{L})$ and $\mathrm{C}$-reactive protein (CRP) $(8.6 \mathrm{mg} / \mathrm{dL})$. Meropenem hydrate ( $0.5 \mathrm{~g} /$ day) was also administered for infectious disease.
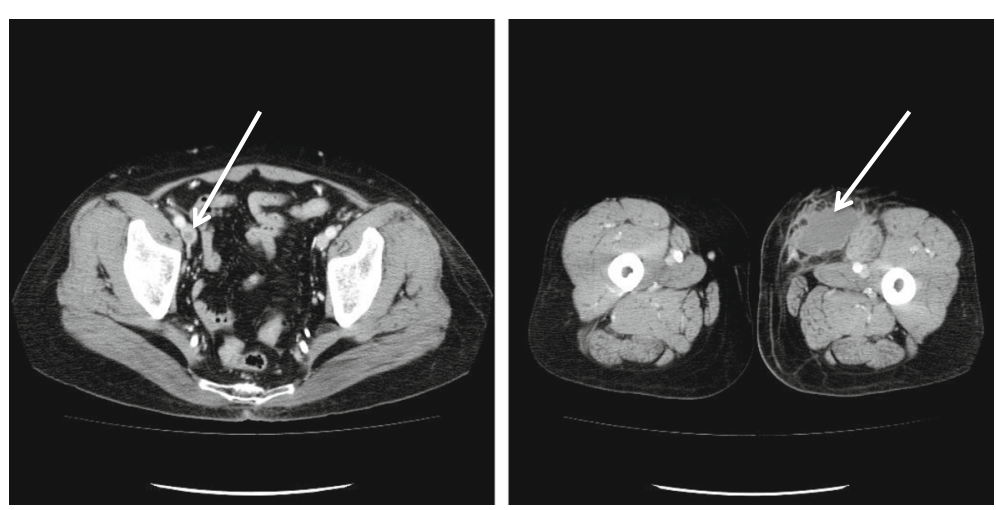

Fig. 1 A contrast-enhanced computed tomography scan revealed (left panel) venous thrombosis in the external iliac vein (arrow) and (right panel) hematoma inside the sartorius muscle and rectus femoris muscle (arrow) (day 3) 
The authors did not place an inferior vena cava filter to protect from new onset of pulmonary embolism attributable to the findings as follows: ultrasonic echocardiography showed no abnormality, and the possibility remained concerning risk of exacerbation of infections by catheterization.

Based on these findings, the patient was diagnosed as oketsu (blood stasis syndrome). Traditional Japanese Kampo medicine, JDI (7.5 g/day: Tsumura, Tokyo, Japan), was administered to improve the symptoms.

Visual analogue scale (VAS) scale in the groin reduced from 85 (day 1) to $33 \mathrm{~mm}$ (day 7), thereafter, $12 \mathrm{~mm}$ (day 12). The subcutaneous hemorrhage disappeared completely on day 16 . The firm mass in the left groin gradually reduced in size and was not palpable on day 19 .

Because laboratory data showed improvement in CRP concentration of less than $0.01 \mathrm{mg} / \mathrm{dL}$, blood culture test remained negative, and clinical symptoms dramatically improved, meropenem hydrate administration was terminated on day 14. A d-dimer concentration also reduced from 13.2 to $0.4 \mu \mathrm{g} / \mathrm{mL}$ on day 14 . The patient underwent successful arteriovenous graft formation in the right forearm on day 15.

The CECT on day 21 revealed that venous thrombosis in the right external iliac vein reduced (Fig. 2, left panel), and hematoma inside the left sartorius muscle and rectus femorius also reduced (Fig. 2, right panel); therefore, the patient was discharged on day 22. The patient did not use analgesic drugs such as NSAIDs throughout the hospitalization.

JDI administration was terminated because no symptoms were observed on day 21 . No adverse reactions to the JDI treatment were observed.

Neither pulmonary embolism nor recurrence of venous thrombosis occurred until 6 months after discharge, thereby, terminating warfarin potassium administration.
At present, the patient is clinically well, showing no signs of exacerbation or relapse of venous thrombosis and subcutaneous hematoma.

\section{Discussion}

The authors found out two important clinical issues in this case. JDI controls the pain associated with subcutaneous hematoma without using analgesics such as NSAIDs. JDI also has an effect of improving the complications associated with venous thrombosis and subcutaneous hematoma caused by percutaneous catheterization in HD patients.

First, the pain was well-controlled by conservative treatment using traditional Japanese Kampo medicine JDI without using analgesics. Several reports in the literature describe the randomized controlled trial comparing the effects on rib fracture between JDI and NSAIDs showing that the treatment period was shorter in JDI than NSAIDs; thereby, JDI is an excellent analgesic from the viewpoint of medical economics [2].

The prescription JDI has been developed by Shuan Kagawa (herbalist), who lived in the eighteenth century, Edo era, Japan [3]. JDI is an herbal mixture that improves the blood circulation in the affected lesion and alleviates contusion-induced swelling and pain. JDI also has been reported to undo the stagnant blood flow and repair blood flow without forming thrombosis. JDI is composed of seven herbs as follows: cinamon bark, cnidium rhizome, nuphar rhizome, quercus bark, glycyrrhiza root, rhubarb rhizome, and clove floral bud [4]. Cnidium rhizome exerts anti-inflammatory and analgesic actions. Nuphar rhizome promotes the resorption of the internal hemorrhage, thereby repairing damaged tissue. Quercus bark has analgesic, anti-inflammatory, detoxyfying, and hemostatic properties. Rhubarb rhizome and clove floral bud improve microcirculation [4]. Several
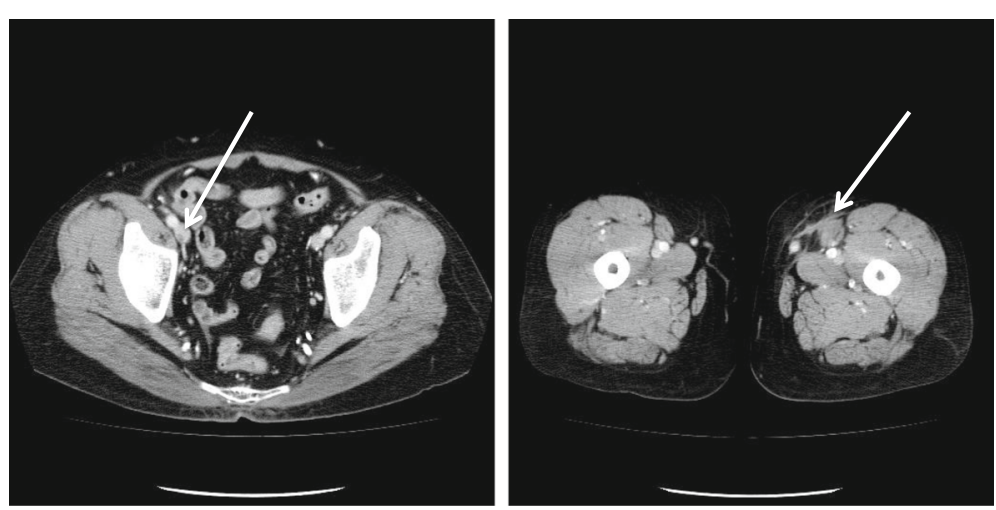

Fig. 2 A contrast-enhanced computed tomography scan revealed that (left panel) venous thrombosis in the external iliac vein reduced compared with day 3 (arrow) and (right panel) hematoma inside the sartorius muscle and rectus femoris muscle also reduced compared with day 3 (arrow) (day 21) 
reports in the literature describe that JDI includes herbs with established antioxidant activity, which may act synergistically when administered as a combination [5-7]. Antioxidant activity has been reported to be involved in the improvement of swelling and pain associated with trauma [8]; therefore, JDI has mainly been used for bruises and sprains in Japan [4]. Nevertheless, no reports were available concerning the effectiveness of JDI in HD patients. The current report indicates the possibility that the pharmacological effect of JDI could be useful for HD patients with catheter-related complications such as pain, thrombosis, and hematoma. Moreover, in this case, JDI was firstly administered as analgesic and was surprisingly effective to resolve thrombosis and subcutaneous hematoma, probably in part, attributable to the effect of cnidium rhizome and quercus bark [4]. It still remains unclear whether JDI administration under the specific condition with renal dysfunction affected the improvement of these catheter-related complications. However, treatment by JDI should be considered as an option for catheterrelated complications among all patients with history of side effects caused by NSAIDs.

In the pathological state, ketsu (blood and its metabolic products that circulate throughout the body) manifests stagnation known as oketsu according to the diagnostic criteria [9]. The major formulations against oketsu for the similar symptoms are as follows: (1) tsudosan, (2) tokakujyokitou, (3) keishibukuryogan, and (4) JDI. To distinguish JDI from other three formulations when prescribing, abdominal symptoms type of sho based on Japanese Kampo medicine show the characteristics as follows: (1) tsudosan, complaint of having stuck sensations in the region beneath the heart; (2) tokakujyokitou, lower abdominal strain appearing accompanied by severe sharp pain on gentle rubbing by fingertip; and (3) keishibukuryogan, pain at side of navel on palpitation. The pain produced at either side of the navel on palpitation is an important abdominal symptom of ketsu congestion. Ketsu-improving agent such as keishibukuryogan may clear up this condition. The present case did not show such abdominal symptoms. No specific abdominal symptoms (type of sho by Kampo diagnosis) exist in the use of JDI [4]; therefore, JDI has been widely used and classified as one of the major drugs improving the pathological state called oketsu in Japanese Kampo medicine.

According to the theory of Japanese Kampo medicine, venous thrombosis and subcutaneous hematoma caused by catheterization are considered as an oketsu condition: a sign of microcirculating disorder. In the present case, pain, thrombosis, and subcutaneous hematoma surprisingly improved with use of JDI in addition to combination therapy with anticoagulant and antibiotic. This could be attributable to that oketsu condition was improved by JDI. The result that the patient had no complication such as thrombosis or coagulation inside the HD circuit also indicates that JDI unlikely inhibits the action of warfarin and heparin. For the treatment of more severe pain, thrombosis, and hematoma by catheterization, it remains unclear which is better either JDI alone therapy or combination therapy with other Kampo oketsuimproving agents such as tsudosan, tokakujyokitou, and keishibukuryogan in addition to JDI. This issue remains to be solved in the future.

Second, femoral vein thrombosis and inguinal hematoma were caused by percutaneous femoral catheterization. The hematoma increased in size and was successfully treated by conservative treatment using traditional Japanese Kampo medicine JDI to resolve oketsu. The venous thrombosis decreased in size after combination therapy with JDI and warfarin potassium.

Because anticoagulants such as heparin are regularly administered in HD treatment, HD patients frequently have complications associated with bleeding. The present case also had a history of side-effects of gastrointestinal bleeding associated with use of NSAIDs, therefore, he was administered JDI. JDI can thus be used safely and economically in HD patients with history of gastrointestinal bleeding caused by NSAIDs.

According to the clinical guidance for the evaluation and the treatment of cardiovascular complications in HD patients by Japanese Society for Dialysis Therapy [10], they state that warfarin potassium for HD patients should be strictly monitored attributable to high risk of bleeding complications such as cerebral hemorrhage in the population [11, 12]. Warfarin potassium significantly increases the risk for bleeding as creatinine clearance. Non-vitamin K-dependent oral anticoagulant agents (NOACs) are currently recommended for patients with atrial fibrillation at risk for stroke instead of warfarin potassium, however, all NOACs are dependent on kidney function for elimination therefore contraindicated in HD population [13]. Anticoagulant therapy for HD patients is difficult accordingly.

If the patient was administered only warfarin potassium as an anticoagulant therapy and meropenem hydrate without JDI, the possibility remains that the pain control might be more difficult, and it might take longer to diminish venous thrombosis and hematoma. Therefore, the quality of life of the patient might be reduced attributable to extension of hospitalization or medication period.

Because JDI contains rhubarb rhizome (laxative) [4], the associated side effects of digestive symptoms such as epigastralgia and diarrhea could be caused. In the present case, the patient did not complain of such symptoms. While taking JDI, the frequency of his bowel movements improved regularly approximately once daily. HD patients overall require water restriction, thereby complicating constipation frequently. Laxative effect of rhubarb rhizome 
may be rather favorable for bowel movement in HD patients.

The favorable indication of JDI in HD patients could be characterized as follows: (1) pain with history of NSAIDs-related gastrointestinal bleeding, (2) complications associated with venous thrombosis or subcutaneous hematoma caused by catheterization, and (3) under treatment with anticoagulants (bleeding tendency). On the other hand, unfavorable indication of JDI in HD patients could be characterized as follows: patients who cannot follow water restriction. Because herbal medicines are generally in granule forms and bitter taste, it requires a certain amount of drinking water at the time of medication. They are more difficult to take than a tablet form; therefore, JDI in capsules or tablets should be desirable in the future. Because the patient accompanied with shivering and high fever, he was administered JDI with use of a jelly specialized for Kampo drugs without drinking water (Rakuraku fukuyaku jelly for Kampo medicine, Ryukakusan, Tokyo, Japan) [14].

JDI can be not only safely used for HD patients with fewer side-effects but also a cheap medicine from the viewpoint of medical economics. Traditional Japanese Kampo and modern medical approaches should be incorporated into the future medical system to exploit the advantage of both medicine. A large number of cases using JDI should be accumulated to maximize the benefits and confirm the excellent effectiveness of JDI in the future.

\section{Conclusions}

The present case indicated as follows: JDI controls the pain associated with hematoma without analgesics such as NSAIDs; JDI also has an effect of improving the complications associated with venous thrombosis and subcutaneous hematoma caused by catheterization in HD patients safely. JDI, one of Kampo drugs, has few side effects and can be used as needed.

\section{Abbreviations}

CECT: Contrast-enhanced computed tomography; CH50: 50\% hemolytic unit factor; CRP: C-reactive protein; HD: Hemodialysis; JDI: Jidabokuippo; NOACs: Non-vitamin K-dependent oral anticoagulant agents; NSAIDs: Nonsteroidal anti-inflammatory drugs; SLE: Systemic lupus erythematosus; VAS: Visual analogue scale

\section{Acknowledgements}

The authors would like to express our sincere thanks to the medical staffs of Nippon Kokan Fukuyama Hospital, Hiroshima, Japan, for collecting samples of the patient and analyzing the samples.

\section{Funding}

Not applicable. Our manuscript does not contain funding.

\section{Authors' contributions}

KW is responsible for the manuscript. KW and YW performed the acquisition and interpretation of data and drafted and revised the manuscript. HAU helped to draft the manuscript. All authors read and approved the final manuscript.

\section{Authors' information}

KW is a Director of the Division of Nephrology and Dialysis, Department of Internal Medicine, Nippon Kokan Fukuyama Hospital, Hiroshima, Japan. HAU is the associate professor of the Department of Chronic Kidney Disease and Cardiovascular Disease, Okayama University Graduate School of Medicine, Dentistry and Pharmaceutical Sciences, Okayama, Japan. YW is a Director of the Division of Nephrology and Dialysis, Department of Internal Medicine, Central Hospital, Hiroshima, Japan.

\section{Competing interests}

The authors declare that they have no competing interests.

\section{Consent for publication}

Written informed consent was obtained from the patient for publication of this case report and the accompanying images.

\section{Ethics approval and consent to participate}

Written informed consent was obtained from the patient for publication of this case report and the accompanying images. This case report was approved by the Ethical Committee of the Nippon Kokan Fukuyama Hospital (IRB approval number: 2016-02) and was conducted in compliance with the Declaration of Helsinki.

\section{Author details}

'Division of Nephrology and Dialysis, Department of Internal Medicine, Nippon Kokan Fukuyama Hospital, 1844, Tsunoshita, Daimon-cho, Fukuyama City, Hiroshima 721-0927, Japan. ${ }^{2}$ Department of Chronic Kidney Disease and Cardiovascular Disease, Okayama University Graduate School of Medicine, Dentistry and Pharmaceutical Sciences, Okayama, Japan. ${ }^{3}$ Division of Nephrology and Dialysis, Department of Internal Medicine, Central Hospital, Hiroshima, Japan.

Received: 15 July 2016 Accepted: 8 December 2016 Published online: 23 January 2017

\section{References}

1. Oliver MJ. Acute dialysis catheters. Semin Dial. 2001;14:432-5.

2. Nakae H, Yokoi A, Kodama H, Horikawa A. Evid Based Complement Alternat Med. 2012:2012:837-958.

3. Asada S. Futsugo-yakushitsu-hokan-kuketsu. Tokyo: Futsugo-yakushitsu 20; 1878 [in Japanese].

4. The Japan Society for Oriental Medicine. Introduction to KAMPO: Japanese traditional medicine. Tokyo: Elsevier Japan K.K; 2005.

5. Nakae $H$, Hebiguchi M, Okuyama M. Jidabokuippo use in patients with fractures of the extremities. Pers Med Univers. 2015:4:66-9.

6. Nakae $\mathrm{H}$. Clinical evaluation of oxidative stress after taking jidabokuippo. Kampo Med. 2010;61:847-52 [in Japanese].

7. Nakae $H$. Determination of the total antioxidant capacity for comparing different types of suspensions of Kampo extract. Int J Integr Med. 2011;3:626 [in Japanese].

8. Povlishock JT, Kontos HA. The role of oxigen radicals in the pathobiology of traumatic brain injury. Hum Cell. 1992;5:345-53.

9. Terasawa K, Shinoda H, Imadaya A, et al. The presentation of diagnositic criteria for "oketsu" syndrome. Kampo Med. 1983;34:1-17 [in Japanese].

10. Japanese Society for Dialysis Therapy. Clinical guidance for the evaluation and the treatment of cardiovascular complications in hemodialysis patients. J Jpn Soc Dial Ther. 2011:44:337-425 [in Japanese].

11. Bleyer AJ, Russell GB, Satko SG. Sudden and cardiac death rates in hemodialysis patients. Kidney Int. 1999;55:1553-9.

12. Reiter MJ, Reiffel JA. Importance of beta blockade in the therapy of serious ventricular arrhythmias. Am J Cardiol. 1991;82:91-191.

13. Chan KE, Giugliano RP, Patel MR, et al. Nonvitamin K anticoagulant agents in patients with advanced chronic kidney disease or on dialysis with AF. J Am Coll Cardiol. 2016;67:2888-99.

14. Wada K. Kampo medicine for dialysis patients. Tokyo: Nakayama Shoten Co., Ltd.; 2005 [in Japanese]. 